\title{
Increase in omega- 6 and decrease in omega- 3 polyunsaturated fatty acid oxidation elevates the risk of exudative AMD development in adults with Chinese diet
}

\author{
Ho Hang Leung ${ }^{\mathrm{a}}$, Alex LK. $\mathrm{Ng}^{\mathrm{b}, \mathrm{c}, 1}$, Thierry Durand ${ }^{\mathrm{d}}$, Ryo Kawasaki ${ }^{\mathrm{e}}$, Camille Oger ${ }^{\mathrm{d}}$, \\ Laurence Balas $^{\mathrm{d}}$, Jean-Marie Galano ${ }^{\mathrm{d}}$, Ian YH. Wong ${ }^{\mathrm{b}, \mathrm{f}, 2}$, Jetty Chung-Yung Lee ${ }^{\mathrm{a}, *}$ \\ ${ }^{\text {a }}$ School of Biological Sciences, The University of Hong Kong, Hong Kong SAR, China \\ ${ }^{\mathrm{b}}$ Department of Ophthalmology, LKS Faculty of Medicine, The University of Hong Kong, Hong Kong SAR, China \\ ${ }^{\mathrm{c}}$ Hong Kong Ophthalmic Associates, Hong Kong SAR, China \\ ${ }^{\mathrm{d}}$ Institut des Biomolécules Max Mousseron, UMR 5247 CNRS, ENSCM, Université de Montpellier, France \\ ${ }^{\mathrm{e}}$ Department of Vision Informatics, Osaka University, Japan \\ ${ }^{\mathrm{f}}$ Department of Ophthalmology, Hong Kong Sanatorium and Hospital, Hong Kong SAR, China
}

\section{A R T I C L E I N F O}

\section{Keywords:}

Macular degeneration

Omega-6/omega-3 polyunsaturated fatty acid

ratio

Carotenoids

Lipid peroxidation

Isoprostanes

Neuroprostanes

4-HNE

\begin{abstract}
A B S T R A C T
Appropriate diet is essential for the regulation of age-related macular degeneration (AMD). In particular the type of dietary polyunsaturated fatty acids (PUFA) and poor antioxidant status including carotenoid levels concomitantly contribute to AMD risk. Build-up of oxidative stress in AMD induces PUFA oxidation, and a mix of lipid oxidation products (LOPs) are generated. However, LOPs are not comprehensively evaluated in AMD. LOPs are considered biomarkers of oxidative stress but also contributes to inflammatory response. In this cross-sectional case-control study, plasma omega-6/omega-3 PUFA ratios and antioxidant status (glutathione, superoxide dismutase and catalase), and plasma and urinary LOPs (41 types) were determined to evaluate its odds-ratio in the risk of developing exudative AMD $(n=99)$ compared to age-gender-matched healthy controls $(n=198)$ in adults with Chinese diet. The odds ratio of developing exudative AMD increased with LOPs from omega-6 PUFA and decreased from those of omega-3 PUFA. These observations were associated with a high plasma omega-6/ omega-3 PUFA ratio and low carotenoid levels. In short, poor PUFA and antioxidant status increased the production of omega-6 PUFA LOPs such as dihomo-isoprostane and dihomo-isofuran, and lowered omega-3 PUFA LOPs such as neuroprostanes due to the high omega-6/omega-3 PUFA ratios; they were also correlated to the risk of AMD development. These findings indicate the generation of specific LOPs is associated with the development of exudative AMD.
\end{abstract}

\section{Introduction}

Age-related macular degeneration (AMD) is an acquired disease of the macula and depicted by progressive visual impairment due to deterioration of the photoreceptor-retinal pigment epithelial complex. It is one of the leading causes of blindness in elderlies of both Western and Asian countries, and the number of patients is expected to increase significantly [1]. The exact pathophysiology of AMD is not fully elucidated, but it is believed that the pathogenesis of AMD is the result of complex multi-factor interactions between metabolic, genetic and environmental factors [2].
Of all the environmental factors, nutrition plays an important role in the prevention and progression of AMD. In the AREDS I and AREDS 2 studies, the only 2 large prospective randomized trials on AMD nutritional interventions showed that supplementation of an antioxidant combination (beta-carotene or lutein/zeaxanthin plus vitamin C, E, zinc and copper) potentially reduced the progression of AMD to advanced level in some patients by $25 \%$ within five years $[3,4]$. It is also proposed lutein/zeaxanthin supplementation may be beneficial to some individuals [4] however, the addition of omega-3 polyunsaturated fatty acid (omega-3 PUFA), namely eicosapentaenoic acid (EPA) and docosahexaenoic acid (DHA) had no effect in lowing the risk of developing

\footnotetext{
* Corresponding author. School of Biological Sciences, The University of Hong Kong Pokfulam Road, Hong Kong Special Administrative Region.

E-mail address: jettylee@hku.hk (J. Chung-Yung Lee).

${ }^{1}$ Co-first Author.

${ }^{2}$ Co-corresponding Author
} 
AMD. These findings contradict to the conclusions of epidemiological studies $[4,5]$, in which DHA is associated with a lower incidence of advanced AMD.

The early sign of AMD onset is characterized by the presence of extracellular deposition, known as drusen, which accumulates between the Burch membrane and retinal pigment epithelium. Previous studies isolated the metabolic debris from AMD patients and detected advanced glycation end products and carboxyethylpyrrole adducts formed from oxidation of fatty acids in the photoreceptor. Indeed, these components $[6,7]$ are PUFA metabolites that are associated to oxidative damage therefore suggests oxidative stress takes part in the pathogenesis and progression of AMD [8].

Oxidative stress generally refers to cellular damage caused by reactive oxygen species (ROS) [9]. Macromolecules in particular lipids, are targets of the damage. Absorbed dietary lipids will be differentially transported to the tissue site to perform their function. Under oxidative stress and inflammation, these lipids become dysfunctional and generate a mix of lipid oxidation products (LOP). Depending on the type of product generated, it may be harmful or beneficial to human health and disease.

It has been found that LOPs derived from PUFA peroxidation is biologically active by participating in various cellular signalling pathways related to pathophysiological conditions [10]. In particular, 15$\mathrm{F}_{2 \mathrm{t}}$-isoprostane (15- $\mathrm{F}_{2 \mathrm{t}}$-IsoP) derived from arachidonic acid (ARA) is not only a hallmark for oxidative stress in vivo, but also known to trigger an excitatory response that are mediated by the thromboxane $\mathrm{A} 2$ receptors in a large number of tissues and vascular systems, including the aorta, carotid, coronary, cerebral, pial, and retinal vasculatures [10-13]. Isoprostanes are also derived from other PUFA, namely $\mathrm{F}_{2}$-dihomoisoprostanes $\left(\mathrm{F}_{2}\right.$-dihomo-IsoPs) from adrenic acid (AdA), $\mathrm{F}_{3}$-isoprostanes $\left(\mathrm{F}_{3}\right.$-IsoPs) from eicosapentaenoic acid (EPA) and $\mathrm{F}_{4}$-neuroprostanes $\left(\mathrm{F}_{4}\right.$-NeuroPs) from docosahexaenoic acid (DHA). Although it is not widely investigated, they are considered to be tissue-specific markers of oxidative stress related to neurological diseases such as Rett Syndrome and epilepsy, but also have potential benefits for cardiovascular health $[10,14,15]$. In addition, LOPs originating from lipoxygenase-mediated PUFA oxidation are largely involved in inflammatory response where those from ARA namely hydroxyeicosatetraenoic acids (HETE) are pro-inflammatory and stimulates platelet activation, while DHA-derived hydroxydocosahexaenoic acids (HDHA) are anti-inflammatory with pro-resolving actions [16].

The breakdown of lipid and formation of an array of LOPs in retinal cells thereby may contribute to the pathophysiology of AMD, while antioxidants like lutein/zeaxanthin and omega-3 PUFAs may modify the oxidation process. However, despite the prompted interest in the role of lipid abnormalities in AMD, previous studies have focused specifically on the role of malondialdehyde and 4-hydroxynonenal (4HNE) [17]. These aldehydes are toxic and potentially form adducts by cross-linking with protein [18]. Nonetheless, information on the role of other LOPs such as isoprostanes of AdA, DPA, DHA and EPA, and different isomers of HETE and HDHA on AMD are insofar not investigated in AMD.

Although AREDS showed the importance of antioxidants and DHA plus EPA in the progression of AMD, the studies were based on nonAsian community [3,4]. Furthermore, oxidative stress status of the patients depended on lipid peroxidation products related to ARA only and not EPA, DHA or other PUFA, that are suggested to have pivotal roles in disease development. We hypothesize that the poor DHA and antioxidant carotenoid diet as reported in our previous study [19] increased the risk of AMD development in adults in part, attributed by certain oxidized products derived from PUFA. In support, we determined (1) the antioxidant status (antioxidant enzymes, carotenoid levels), (2) plasma omega-6 PUFA to omega-3 PUFA ratio status and (3) plasma and urine lipid oxidation products (isoprostanes, HETE, HDHA, specialized pro-resolving mediators, aldehydes) and 8-hydroxy-2'deoxyguanosisne (a DNA adduct of oxidative damage) of AMD patients compared to healthy age-matched controls in adults with Chinese diet.

\section{Materials and methods}

\subsection{Chemicals and materials}

All organic solvents were at least analytical grade and purchased from Sigma-Aldrich (St. Louis, Mo, USA). All isoprostanoids, HDHAs, HETEs, 4-hydroxyhexenal (4-HHE), 4-HNE, and resolvin standards were purchased from Cayman Chemical Co. (Ann Arbor, MI, USA); 8Hydroxy-2'-deoxyguanosine (8-OHdG) was purchased from SigmaAldrich; neuroprotectin (NPD1), dihomo-IsoPs, dihomo-isofurans (dihomo-IsoFs) and NeuroPs were synthesized by the Institut des Biomolécules Max Mousseron (IBMM, Montpellier, France) as previously described [20-24]. Glutathione (GSH), superoxide dismutase (SOD), catalase (CAT) and bicinchoninic acid (BCA) assay kits were all purchased from Cayman Chemical Co.

\subsection{Study design}

This is a cross-sectional case-control study conducted at the Queen Mary Hospital in Hong Kong and the Grantham Hospital in Hong Kong from December 2015 to December 2017 for a period of two years. The study was approved by the University of Hong Kong Institutional Review Board/Hospital Authority Hong Kong West Cluster (No. UW 14-405) and complies with the principles of the Helsinki Declaration. All patients signed informed consent before joining the study. Detailed recruitment criteria and baseline characteristics of the subjects are described in previous study [19]. Plasma fatty acids and carotenoid levels were measured [19] and reported as total carotenoids and omega-6/omega-3 PUFA ratios in this study. These values were not calculated previously [19].

\subsection{Measurement of PUFA oxidation products}

The plasma and urine samples were prepared and quantified using liquid chromatography quadrupole time-of-flight tandem mass spectrometry (LC-QTOF-MS/MS) method described [25] with modification. In brief, plasma samples were thawed on ice and centrifuged at $3000 \mathrm{x} \mathrm{g}$ for $10 \mathrm{~min}$ at $4{ }^{\circ} \mathrm{C}$ to remove denatured proteins. A volume of $200 \mu \mathrm{L}$ plasma was added to $10 \mathrm{~mL}$ Folch solution for lipid extraction. After phase separation, the lipid-containing lower layer of chloroform was taken and dried under nitrogen gas. The sample was resuspended with deuterated internal standard mixture $(0.1 \mathrm{ng} / \mu \mathrm{L}$ in methanol). Afterwards, $1 \mathrm{~N}$ potassium hydroxide in methanol was added for alkaline hydrolysis, and then neutralized with $5 \mathrm{~N}$ hydrochloric acid. The oxidized PUFA products were extracted by mixed anion solid phase extraction (SPE, MAX Waters, USA). The SPE column was first conditioned with methanol and $20 \mathrm{mM}$ formic acid. After loading the sample, it was washed with $2 \%$ ammonium hydroxide and $20 \mathrm{mM}$ formic acid. Then, the final elute hexane/ethanol/acetic acid (v/v/v; 70/29.4/0.6) was collected. The elute was dried at $37^{\circ} \mathrm{C}$ under nitrogen gas and resuspended in $100 \mu \mathrm{L}$ methanol for immediate analysis.

Sciex X500R QTOF system (Sciex Applied Biosystems, MA, USA) coupled to an Exion LC AC liquid chromatograph with C18 column (150 x $2.1 \mathrm{~mm}, 2.6 \mu \mathrm{m}$ particle size, Phenomenex, USA) maintained at $40^{\circ} \mathrm{C}$ was used in the analysis. The flow rate was set to $300 \mu \mathrm{L} / \mathrm{min}$ and the injection volume was $10 \mu \mathrm{L}$. A mobile phase consisting of $0.1 \%$ aqueous acetic acid (A) and $0.1 \%$ acetic acid/methanol (B) was used for LC. The gradient conditions were set at $20 \%$ of solvent B for $2 \mathrm{~min}$, increased from $20 \%$ to $98 \%$ within $8 \mathrm{~min}$ and then held for $5 \mathrm{~min}$. Thereafter, solvent B was returned to $20 \%$ in $1 \mathrm{~min}$ and held for another $5 \mathrm{~min}$. The QTOF system was operated in negative electrospray ionization (ESI) mode. The spray voltage was set to $-4500 \mathrm{~V}$ and nitrogen was used as the curtain gas. The temperature of the ionization chamber was set at $350^{\circ} \mathrm{C}$, and the ion source gases 1 and 2 were maintained at 35 and 45 
psi, respectively. For TOF MS, the declustering potential (DP) was $-80 \mathrm{eV}$ and the collision energy (CE) was $-10 \mathrm{eV}$. The scan mode was set as multiple reaction monitoring (MRM).

A total of 39 LOPs (chromatogram, $\mathrm{m} / \mathrm{z}$ of the precursor and daughter, DP and CE ions are found in Tables S1 and S2 in the Supplementary Material 1) were evaluated in this study, of which 37 products were within the quantitative range. The quantification of each analyte was determined by correlating the peak area to its corresponding deuterated internal standard peak. For analytes without corresponding deuterated internal standard, the following deuterated internal stan-

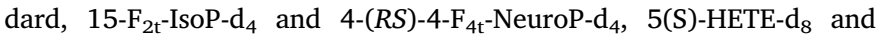
DHA- $d_{5}$, were used for quantification. Calibration curves were set up over a concentration range of $0.01-10 \mathrm{ng} / \mu \mathrm{L}$. Each curve point was determined in triplicate and the regression coefficient ranged between 0.933 - 0.998. For urine samples, the LOP concentrations were normalized with creatinine levels and expressed as $\mu \mathrm{g} / \mathrm{mg}$ creatinine.

\subsection{Measurement of 4-hydoxynonenal and 4-hydroxyhexenal}

To $100 \mu \mathrm{L}$ plasma or $1 \mathrm{~mL}$ urine samples, $200 \mu \mathrm{L}$ of BHT solution $\left(1 \mathrm{mg} / \mathrm{mL}\right.$ in ethanol), $100 \mu \mathrm{L}$ of internal standard mix containing $d_{3^{-}}$ $\mathrm{HHE}$ and $\mathrm{d}_{3}-\mathrm{HNE}(0.25 \mathrm{ng} / \mu \mathrm{L}$ in ethanol) and $2200 \mu \mathrm{L}$ of ethanol-water (50:50 v/v) were added. The sample vortexed for $1 \mathrm{~min}$ before centrifuged at $2100 \mathrm{x} \mathrm{g}$ for $10 \mathrm{~min}$. A volume of $2 \mathrm{~mL}$ of the supernatant was collected, filtered with a hydrophilic filter, and transferred to a new test-tube with a cap.

Dinitrophenylhydrazone (DNPH) derivatization was performed by adding $2 \mathrm{~mL} 0.05 \mathrm{M}$ DNPH solution in acetonitrile/acetic acid $(9: 1 \mathrm{v} / \mathrm{v})$ into $2 \mathrm{~mL}$ of the extract. The tubes were incubated in $60^{\circ} \mathrm{C}$ water bath for $2 \mathrm{~h}$. After derivatization, $2 \mathrm{~mL}$ of water and $2 \mathrm{~mL}$ of hexane were added to the solution to collect the derivatives. The extraction was repeated with another $2 \mathrm{~mL}$ hexane. The solvents were pooled together and evaporated at $37{ }^{\circ} \mathrm{C}$ under nitrogen. The dried extract was resuspended with $100 \mu \mathrm{L}$ of $0.1 \%$ acetic acid/acetonitrile (60:40 v/v).

The separation and quantification of 4-HHE and 4-HNE were performed using an ExionLC ${ }^{\mathrm{TM}}$ AC analytical HPLC, coupled with a Sciex X500R QTOF System. The HPLC system was connected with Kinetex C18 column ( $150 \times 2.1 \mathrm{~mm}, 2.6 \mathrm{um}$, Phenomenex, USA) and warmed to $40{ }^{\circ} \mathrm{C}$ to achieve stable separation. The mobile phases are $0.1 \%$ acetic acid in water (A) and $0.1 \%$ acetic acid in methanol (B), with a flow rate of $0.3 \mathrm{~mL} / \mathrm{min}$. A gradient elution was developed as follow: $40 \%$ to $65 \%$ $\mathrm{B}$ for the first $8.5 \mathrm{~min}$, gradual increase to $100 \% \mathrm{~B}$ in $4 \mathrm{~min}$ and maintain for $7.5 \mathrm{~min}$, then decrease to $40 \% \mathrm{~B}$ in $2 \mathrm{~min}$ and maintain for $6 \mathrm{~min}$ reconditioning. The analysis with the mass spectrometer was performed in $\mathrm{MRM}^{\mathrm{HR}}$ mode, with negative electrospray ionization at a source temperature of $500^{\circ} \mathrm{C}$. Quantification was made by by correlating the peak area to its corresponding deuterated internal standard peak.

\subsection{Measurement of DNA oxidation products}

A DNA adduct, 8-hydroxy-2'-deoxyguanosine (8OHdG) was determined in plasma and urine samples. The samples were prepared and determined as described by Hu et al. method [26] using LC-QTOF-MS/ MS (X500R Sciex Applied Biosystems, MA, USA).

\subsection{GSH, SOD, CAT, BCA and creatinine assay kits}

The plasma samples were prepared and measured as described in the user manuals. BCA levels in plasma samples were used for normalization of GSH, SOD and CAT values ( $\mathrm{n}=20$ for both control and AMD group); creatinine levels were used for normalization of LOPs in urine samples.

\subsection{Statistical analysis}

All statistical methods were performed by IBM $^{\circledR}$ SPSS $^{\circledast}$ Statistics (version 25.0 for Windows, Armonk, NY USA) and the data is expressed as mean (standard deviation). The concentration of LOPs between AMD group and healthy control group was compared using an independent $t$ test. Conditional logistic regression model was also used to adjust for the multiple variables to calculate the odds ratios (ORs) of having AMD and the level of each LOP in the plasma. Pearson correlation was performed to study the relationship between total carotenoids, omega-6/ omega-3 PUFA ratio and LOP. A $p$-value of 0.05 or less were considered statistically significant.

\section{Results}

As previously described [19], a total of 99 exudative AMD patients and 198 age- and gender-matched controls were required to meet the number of sampling criteria for the study. The mean age was 73.7 (10.2) years in AMD and the control group was 67.1 (9.3). There was no significant difference in BMI between the healthy control group $\left(24.48 \pm 3.81 \mathrm{~kg} / \mathrm{m}^{2}\right)$ and AMD $\left(24.17 \pm 3.22 \mathrm{~kg} / \mathrm{m}^{2}\right)$.

As shown, patients with exudative AMD had lower total carotenoid (lutein + zeaxanthin + lycopene + beta-carotene) levels compared to healthy control. The omega-6/omega-3 PUFA ratio in the healthy control group was approximately 8 to 1 , but significantly higher in the AMD group where a ratio of 11 to 1 approximately was recorded (Table 1).

Due to limited number of plasma samples, only 20 samples from each AMD group and healthy control group were tested for antioxidant related enzymes (GSH, SOD, catalase). The group size was based on our pilot study as described in the previous report [19]. There was no difference in GSH levels between the groups (Fig. 1A). On the other hand, SOD (Fig. 1B) and catalase (Fig. 1C) were both significantly higher in the AMD group.

A range of LOPs derived from PUFAs were measured in plasma and urine samples. The profiles were noticeably different between AMD and healthy control groups. Especially in the AMD group, the plasma levels of ARA-derived 5- $\mathrm{F}_{2 \mathrm{t}}$-IsoP, 15- $\mathrm{F}_{2 \mathrm{t}}$-IsoP, 5-HETE and 11-HETE were significantly higher (Table 2). The concentration of their urinary $15-\mathrm{F}_{2 \mathrm{t}^{-}}$ IsoP and its down-stream metabolite 2,3-dinor-5,6-dihydro- $15-\mathrm{F}_{2 \mathrm{t}}$-IsoP also showed to be elevated. Consistently, levels of $4-\mathrm{F}_{3 \mathrm{t}}$-IsoP from omega-6 docosapentaenoic acid (DPA) and 17-dihomo- $\mathrm{F}_{2 \mathrm{t}}$-IsoP from AdA was significantly higher in both plasma and urine for AMD compared to control, while 17-dihomo- $\mathrm{F}_{2 \mathrm{t}}$-IsoF from AdA was elevated only in the urine. Other omega- 6 PUFA oxidation products were similar levels between AMD group and the control group.

On the other hand, the urinary level of EPA-derived $8-\mathrm{F}_{3 \mathrm{t}}$-IsoP and resolvin E1 (RvE1) in plasma were lower in the AMD group compared to healthy control group (Table 3). Significantly lower plasma concentrations of DHA-derived 14-HDHA, 17-HDHA, resolvin D1 (RvD1), $\mathrm{NPD} 1$, and NeuroPs including 4- $\mathrm{F}_{4 \mathrm{t}^{-}}$-NeuroP, $10-\mathrm{F}_{4 \mathrm{t}^{-}}$-NeuroP, $13-\mathrm{F}_{4 \mathrm{t}^{-}}$ NeuroP and $20-\mathrm{F}_{4 \mathrm{t}}$-NeuroP were also observed. The urinary levels of 4-

Table 1

Plasma carotenoids levels and omega-6/omega-3 PUFA ratios of AMD group and control group.

\begin{tabular}{llll}
\hline & & Mean (SD) & $\begin{array}{l}\text { 95\% Confidence } \\
\text { Interval }\end{array}$ \\
\hline Total carotenoids (mg/L) & Controls & $2.89(1.64)$ & $2.66-3.12$ \\
& AMD & $1.44(0.73)$ & $1.29-1.60$ \\
& & $* * *$ & \\
omega-6/omega-3 PUFA & Controls & $8.26(2.86)$ & $7.86-10.33$ \\
$\quad$ ratio & AMD & $11.11(3.91)$ & $8.66-11.89$ \\
& & $* * * *$ & \\
\hline
\end{tabular}

Total carotenoids is the sum of lutein + zeaxanthin + lycopene + beta-carotene. PUFA: polyunsaturated fatty acid. ${ }^{* * *} \mathrm{p}<0.001$ and $* * * \mathrm{p}<0.0001$ indicates statistical significance between AMD patients $(\mathrm{n}=99)$ and healthy control subjects $(\mathrm{n}=198)$. 
A

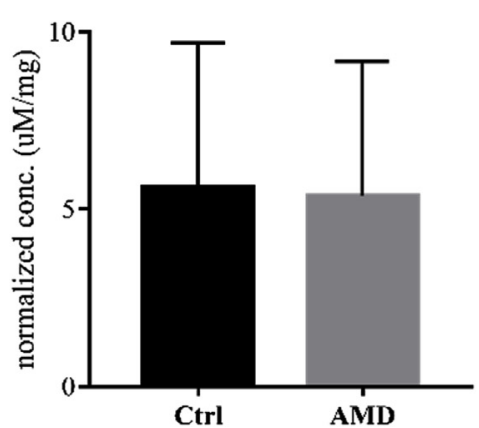

B

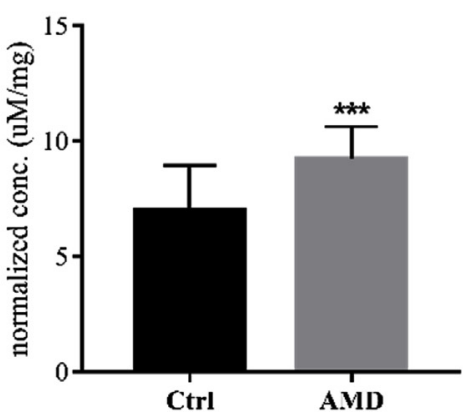

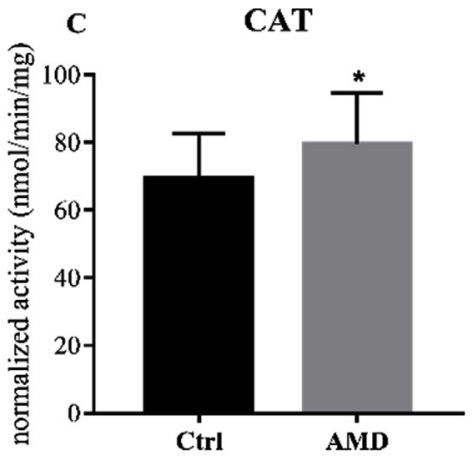

Fig. 1. Antioxidant enzymes determined in plasma. (A) glutathione (GSH) concentration, (B) superoxide dismutase (SOD) concentration and (C) catalase (CAT) activity of AMD group and healthy control (Ctrl) group. Column bars are mean (S.D.). *p $<0.05$, ${ }^{* * *} \mathrm{p}<0.001$ AMD vs Ctrl.

$\mathrm{F}_{4 \mathrm{t}}$-NeuroP, $10-\mathrm{F}_{4 \mathrm{t}}$-NeuroP and $14-\mathrm{F}_{3 \mathrm{t}}$-IsoP from omega-3 DPA were consistently lower in AMD group compared to healthy control group. Other omega-3 PUFA oxidation products levels were similar between the AMD group and the healthy control group.

Aside from PUFA oxidation, oxidative damage of the DNA as measured by 8-OHdG was significantly higher in the AMD group compared to healthy control group in both plasma and urine (Fig. 2).

Using logistic regression analysis, specific LOPs in both plasma and urine have been identified as protective and risk factors of exudative AMD. It was found that $5-\mathrm{F}_{2 \mathrm{t}}$-IsoP, 17-dihomo- $\mathrm{F}_{2 \mathrm{t}}$-IsoP, 4- $\mathrm{F}_{3 \mathrm{t}}$-IsoP and particularly, 4-HNE and 8-OHdG in plasma were associated with higher risk of developing exudative AMD (Table 4) whereas 2,3-dinor-5,6-dihydro-15- $\mathrm{F}_{2 \mathrm{t}}$-IsoP, 4-HNE, 4- $\mathrm{F}_{3 \mathrm{t}}$-IsoP, 17-dihomo- $\mathrm{F}_{2 \mathrm{t}}$-IsoF, 8-OHdG, and particularly, 11-HETE in urine indicated higher AMD risk (Table 5). In contrast, higher levels of 10- $\mathrm{F}_{4 \mathrm{t}}$-NeuroP, 20- $\mathrm{F}_{4 \mathrm{t}}$-NeuroP, 14-HDHA, $17-$ HDHA, NPD1 and RvE1 in plasma and urinary $4-\mathrm{F}_{4 \mathrm{t}^{-}}-\mathrm{NeuroP}, 10-\mathrm{F}_{4 \mathrm{t}^{-}}$ NeuroP, 8- $\mathrm{F}_{3 \mathrm{t}}$-IsoP are associated to lower AMD risk.

Pearson correlation was tested to determine the relationship between nutrition status i.e., total carotenoids and omega-6/omega-3 PUFA ratio, and antioxidant status (GSH, SOD, CAT) of the AMD patients. There was a strong, positive correlation between omega-6/ omega-3 PUFA ratio and SOD $(r=0.45, n=40, p=0.004)$, while a negative correlation between omega-6/omega-3 PUFA ratio and GSH ( $r=-0.38, n=40, p=0.036)$ was also observed. Interestingly, there was a negative correlation between omega-6/omega-3 PUFA ratio and total carotenoid levels $(\mathrm{r}=-0.18, \mathrm{n}=286, \mathrm{p}=0.002)$.

When applying the Pearson correlation to determine relationship between nutrition status and oxidative damage (lipid oxidation, DNA oxidation), it showed that levels of 5-HETE, 4-HNE and 8-OHdG were negatively correlated to total carotenoid levels (Table 6). There was also a strong, negative correlation between NeuroP and RvD1 concentrations with omega-6/omega-3 PUFA ratio. Moreover, levels of 15$\mathrm{F}_{2 \mathrm{t}}$-IsoP, 2,3-dinor- $15-\mathrm{F}_{2 \mathrm{t}}$-IsoP, 17-dihomo- $\mathrm{F}_{2 \mathrm{t}}$-IsoP and 4- $\mathrm{F}_{3 \mathrm{t}}$-IsoP were found to be positively related with omega-6/omega-3 PUFA ratio.

\section{Discussion}

It is acknowledged that lifestyle factors, especially eating habits, have a significant effect on the pathogenesis of AMD [2]. However, most of the research to date, emphasized on the effect of supplements,

Table 2

Concentration of omega-6 PUFA oxidation products in plasma and urine samples of AMD group and healthy control group.

\begin{tabular}{|c|c|c|c|c|}
\hline & \multicolumn{2}{|c|}{ Healthy control $(\mathrm{n}=198)$} & \multicolumn{2}{|l|}{$\operatorname{AMD}(n=99)$} \\
\hline & Plasma (ng/mL) & Urine $(\mu \mathrm{g} / \mathrm{mg} \mathrm{Cr})$ & Plasma (ng/mL) & Urine $(\mu \mathrm{g} / \mathrm{mg} \mathrm{Cr})$ \\
\hline \multicolumn{5}{|c|}{ Arachidonic acid derived } \\
\hline $5-\mathrm{F}_{2 \mathrm{t}}-\mathrm{IsoP}$ & $29.63(17.07)$ & $3.96(2.77)$ & $39.61(24.46) * * *$ & $3.92(3.47)$ \\
\hline $15-\mathrm{F}_{2 \mathrm{t}}$-IsoP & $69.55(45.78)$ & $39.80(26.52)$ & $108.8(66.74) * * *$ & $47.09(20.65) * *$ \\
\hline Dinor-15- $\mathrm{F}_{2 \mathrm{t}}$-IsoP & & $3.58(2.56)$ & & $3.62(2.45)$ \\
\hline Dihydro-15- $\mathrm{F}_{2 \mathrm{t}}$-IsoP & & $3.76(2.34)$ & & $4.63(2.23) * *$ \\
\hline Tetranor-15- $\mathrm{F}_{2 \mathrm{t}}$-IsoP & & $3.83(2.57)$ & & $3.75(2.56)$ \\
\hline $\mathrm{PGF}_{2 \alpha}$ & $44.41(30.85)$ & $3.68(2.59)$ & $43.56(30.5)$ & $3.82(2.85)$ \\
\hline 5-HETE & $24.28(13.47)$ & $1.02(0.59)$ & $30.39(27.74) * *$ & $0.91(0.60)$ \\
\hline 8-HETE & $40.77(9.58)$ & $4.02(2.56)$ & $42.28(31.74)$ & $3.87(1.86)$ \\
\hline 9-HETE & $37.66(20.91)$ & $0.50(0.30)$ & $33.72(23.28)$ & $0.44(0.24)$ \\
\hline 11-HETE & 33.45 (23.29) & $0.89(0.51)$ & $40.98(29.60) *$ & $1.01(0.67)$ \\
\hline 12-HETE & $30.66(21.70)$ & $0.70(0.44)$ & $35.54(24.05)$ & $0.64(0.38)$ \\
\hline 15-HETE & $45.38(26.52)$ & $0.82(0.49)$ & $49.04(23.24)$ & $0.72(0.51)$ \\
\hline 20-HETE & $44.41(27.34)$ & $2.86(2.41)$ & $41.08(23.95)$ & $2.35(1.44)$ \\
\hline \multicolumn{5}{|l|}{ Adrenic acid derived } \\
\hline 7-dihomo-F ${ }_{2 t}$-IsoP & $45.59(40.53)$ & $0.38(0.24)$ & $44.30(27.16)$ & $0.39(0.24)$ \\
\hline 17-dihomo- $\mathrm{F}_{2 \mathrm{t}}$-IsoP & $21.41(13.37)$ & $0.89(0.69)$ & $34.73(25.45) * * *$ & $1.15(0.82) *$ \\
\hline 7-dihomo- $\mathrm{F}_{2 \mathrm{t}}$-IsoF & $7.01(4.84)$ & $0.77(0.60)$ & $6.77(3.86)$ & $0.73(0.45)$ \\
\hline 17-dihomo- $\mathrm{F}_{2 \mathrm{t}}$-IsoF & 36.84 (26.09) & $0.66(0.45)$ & $41.48(22.83)$ & $0.81(0.49) *$ \\
\hline \multicolumn{5}{|c|}{ Omega-6 Docosapentaenoic acid derived } \\
\hline $4-\mathrm{F}_{3 \mathrm{t}}-\mathrm{IsoP}$ & $39.83(32.66)$ & $0.73(0.58)$ & $50.96(35.77) * *$ & $1.02(0.78) * *$ \\
\hline \multicolumn{5}{|c|}{ Peroxidation end product } \\
\hline 4-HNE & $5.62(2.71)$ & $2.67(1.94)$ & $12.76(4.55)$ & $3.78(2.75)$ \\
\hline
\end{tabular}

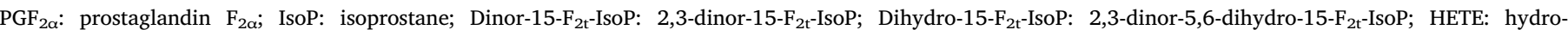

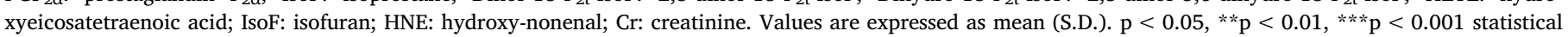
significance between AMD patients and healthy control subjects. 
Table 3

Concentration of omega-3 PUFA oxidation products in plasma and urine samples of AMD group and control group.

\begin{tabular}{|c|c|c|c|c|}
\hline & \multicolumn{2}{|c|}{ Healthy control $(\mathrm{n}=198)$} & \multicolumn{2}{|l|}{ AMD (n = 99) } \\
\hline & $\begin{array}{l}\text { Plasma (ng/ } \\
\text { mL) }\end{array}$ & $\begin{array}{l}\text { Urine }(\mu \mathrm{g} / \mathrm{mg} \\
\mathrm{Cr})\end{array}$ & $\begin{array}{l}\text { Plasma (ng/ } \\
\text { mL) }\end{array}$ & $\begin{array}{l}\text { Urine }(\mu \mathrm{g} / \mathrm{mg} \\
\mathrm{Cr})\end{array}$ \\
\hline \multicolumn{5}{|c|}{ Eicosapentaenoic acid derived } \\
\hline $5-\mathrm{F}_{3 \mathrm{t}}$-IsoP & $38.02(24.31)$ & $3.52(2.35)$ & $40.74(23.17)$ & $3.24(2.40)$ \\
\hline $8-\mathrm{F}_{3 \mathrm{t}}$-IsoP & 38.10 (21.97) & $0.56(0.39)$ & $39.75(23.26)$ & $\begin{array}{l}0.35(0.26) \\
* * *\end{array}$ \\
\hline $18-\mathrm{F}_{3 \mathrm{t}}$-IsoP & $96.15(83.31)$ & $3.94(2.62)$ & $100.20(82.15)$ & $4.03(2.38)$ \\
\hline RvE1 & $4.36(2.63)$ & & $3.45(2.93) * *$ & \\
\hline \multicolumn{5}{|c|}{ Docosahexaenoic acid derived } \\
\hline $4-\mathrm{F}_{4 \mathrm{t}}-\mathrm{NeuroP}$ & $41.61(22.88)$ & $1.98(1.62)$ & $\begin{array}{l}34.35(18.47) \\
* *\end{array}$ & $1.01(0.79)$ \\
\hline $10-\mathrm{F}_{4 \mathrm{t}}$-NeuroP & $45.26(24.30)$ & $1.38(1.14)$ & $\begin{array}{l}32.45(15.18) \\
* * *\end{array}$ & $\begin{array}{l}0.43(0.31) \\
* * * *\end{array}$ \\
\hline $13-\mathrm{F}_{4 \mathrm{t}}-\mathrm{NeuroP}$ & $4.29(2.82)$ & & $\begin{array}{l}2.36(1.70) \\
* * * *\end{array}$ & \\
\hline $14-\mathrm{F}_{4 \mathrm{t}}-\mathrm{NeuroP}$ & $21.03(10.48)$ & & $18.79(12.16)$ & \\
\hline $20-\mathrm{F}_{4 \mathrm{t}}$-NeuroP & $9.76(6.76)$ & & $\begin{array}{l}5.53(2.79) \\
* * * *\end{array}$ & \\
\hline RvD1 & $6.89(4.52)$ & & $5.07(2.33) * * *$ & \\
\hline NPD1 & 34.54 (23.39) & & $28.60(17.95)$ * & \\
\hline 4-HDHA & $28.14(23.75)$ & & $23.92(13.94)$ & \\
\hline 7-HDHA & $29.76(21.80)$ & & 34.77 (21.57) & \\
\hline 8-HDHA & $40.01(26.62)$ & & $36.81(23.96)$ & \\
\hline 11-HDHA & $3.36(2.45)$ & & $3.25(1.87)$ & \\
\hline 14-HDHA & $40.65(28.04)$ & & $\begin{array}{l}24.95(15.26) \\
* * * *\end{array}$ & \\
\hline 17-HDHA & $37.47(18.25)$ & & $30.83(15.6) * *$ & \\
\hline \multicolumn{5}{|c|}{ Omega-3 Docosapentaenoic acid derived } \\
\hline $14-\mathrm{F}_{3 \mathrm{t}}$-IsoP & $4.63(2.55)$ & $0.95(0.69)$ & $3.50(2.57)$ & $0.71(0.49)$ * \\
\hline \multicolumn{5}{|c|}{ Peroxidation end product } \\
\hline 4-HHE & $50.44(11.72)$ & $3.95(2.94)$ & $49.84(12.48)$ & $3.61(2.09)$ \\
\hline
\end{tabular}

IsoP: isoprostane; NeuroP: neuroprostane; RvD: resolvin D; RvE: resolvin E; HDHA: hydroxy-docosahexaenoic acid; HHE: hydroxy-hexenal; Cr: creatinine. Values are expressed as mean (S.D.). ${ }^{*} \mathrm{p}<0.05$, ${ }^{* *} \mathrm{p}<0.01,{ }^{* * *} \mathrm{p}<0.001$, $* * * * \mathrm{p}<0.0001$ statistical significance between AMD patients and healthy control subjects.
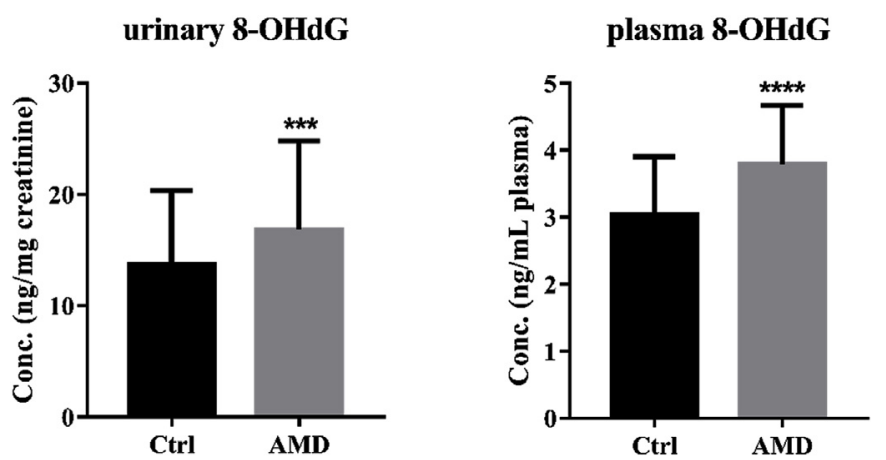

Fig. 2. Concentration of urinary and plasma 8-hydroxy-2'-deoxyguanosine (8OHdG) of AMD group and healthy control group. Column bars are mean (S.D.) $* * * \mathrm{p}<0.001, * * * * \mathrm{p}<0.0001$ statistical significance between AMD patients ( $\mathrm{n}=99)$ and healthy control subjects $(\mathrm{n}=198)$.

and few studies have focused on the relationship between circulating nutrients (including fatty acids and carotenoids) and AMD. Furthermore, most observations on PUFA and carotenoid supplements are based in Caucasian population with western diet [27,28]. There is little data on whether these supplements are equally relevant in reducing AMD risks in Asian populations, where dietary lipid intake is different from Western populations.

In our previous study [19], patients with exudative AMD in Hong Kong Chinese population were found to have lower plasma carotenoids and omega-3 PUFA, in particular EPA, DHA and $\alpha$-linolenic acid (ALA)
Table 4

Odds ratio of plasma PUFA oxidation products of being an AMD in the sample population. Only those with statistical significance are shown.

\begin{tabular}{|c|c|c|c|c|}
\hline & Odds Ratio & Standard error & $p$-value & $95 \% \mathrm{CI}$ \\
\hline \multicolumn{5}{|c|}{ Arachidonic acid-derived } \\
\hline $5-\mathrm{F}_{2 \mathrm{t}}$-IsoP & 1.041 & 0.020 & 0.046 & $1.001-1.083$ \\
\hline \multicolumn{5}{|l|}{ Adrenic acid-derived } \\
\hline 17-dihomo- $\mathrm{F}_{2 \mathrm{t}}$-IsoP & 1.163 & 0.043 & $<0.0001$ & $1.070-1.265$ \\
\hline \multicolumn{5}{|c|}{ Docosapentaenoic acid-derived } \\
\hline $4-\mathrm{F}_{3 \mathrm{t}}-\mathrm{IsoP}$ & 1.042 & 0.015 & 0.006 & $1.012-1.072$ \\
\hline \multicolumn{5}{|c|}{ Eicosapentaenoic acid-derived } \\
\hline RvE1 & 0.810 & 0.095 & 0.026 & $0.672-0.975$ \\
\hline \multicolumn{5}{|c|}{ Docosahexaenoic acid-derived } \\
\hline $10-\mathrm{F}_{4 \mathrm{t}}-\mathrm{NeuroP}$ & 0.962 & 0.014 & 0.007 & $0.936-0.989$ \\
\hline $20-\mathrm{F}_{4 \mathrm{t}}-\mathrm{NeuroP}$ & 0.756 & 0.082 & 0.001 & $0.644-0.887$ \\
\hline 14-HDHA & 0.969 & 0.012 & 0.006 & $0.947-0.991$ \\
\hline 17-HDHA & 0.955 & 0.016 & 0.003 & $0.926-0.985$ \\
\hline NPD1 & 0.969 & 0.013 & 0.018 & $0.944-0.995$ \\
\hline \multicolumn{5}{|c|}{ Peroxidation end product } \\
\hline 4-HNE & 2.509 & 0.185 & $<0.0001$ & $1.747-3.604$ \\
\hline \multicolumn{5}{|l|}{ DNA oxidation } \\
\hline 8-OHdG & 2.732 & 0.172 & $<0.0001$ & $1.929-3.830$ \\
\hline
\end{tabular}

Odds ratio adjusted for age and gender. CI: confidence interval; IsoP: isoprostane; NeuroP: neuroprostane; HDHA: hydroxy-docosahexaenoic acid; RvE: resolvin E; NPD: neuroprotectin; HNE: hydroxy-nonenal; 8-OHdG: 8-hydroxy2'deoxy-guanosine.

Table 5

Odds ratio of urinary oxidation products of being an AMD in the sample population. Only those with statistical significance are shown.

\begin{tabular}{|c|c|c|c|c|}
\hline & Odds Ratio & Standard error & $p$-value & $95 \% \mathrm{CI}$ \\
\hline \multicolumn{5}{|c|}{ Arachidonic acid-derived } \\
\hline Dihydro-15- $\mathrm{F}_{2 \mathrm{t}}$-IsoP & 1.024 & 0.009 & 0.011 & $1.005-1.043$ \\
\hline 11-HETE & 9.551 & 0.816 & 0.006 & $1.930-47.275$ \\
\hline \multicolumn{5}{|l|}{ Adrenic acid-derived } \\
\hline 17-dihomo- $\mathrm{F}_{2 \mathrm{t}}$-IsoF & 4.725 & 0.479 & 0.001 & $1.850-12.069$ \\
\hline \multicolumn{5}{|c|}{ Docosapentaenoic acid-derived } \\
\hline $4-\mathrm{F}_{3 t}-$ IsoP & 2.430 & 0.327 & 0.007 & $1.281-4.610$ \\
\hline \multicolumn{5}{|c|}{ Eicosapentaenoic acid-derived } \\
\hline $8-\mathrm{F}_{3 \mathrm{t}}-\mathrm{IsoP}$ & 0.159 & 0.748 & 0.014 & $0.037-0.688$ \\
\hline \multicolumn{5}{|c|}{ Docosahexaenoic acid-derived } \\
\hline $4-\mathrm{F}_{4 \mathrm{t}}-\mathrm{NeuroP}$ & 0.554 & 0.224 & 0.008 & $0.357-0.859$ \\
\hline $10-\mathrm{F}_{4 \mathrm{t}}-\mathrm{NeuroP}$ & 0.102 & 0.540 & $<0.0001$ & $0.035-0.294$ \\
\hline \multicolumn{5}{|c|}{ Peroxidation end product } \\
\hline 4-HNE & 1.314 & 0.096 & 0.004 & $1.089-1.586$ \\
\hline \multicolumn{5}{|l|}{ DNA oxidation } \\
\hline 8-OHdG & 1.072 & 0.020 & $<0.0001$ & $1.031-1.115$ \\
\hline
\end{tabular}

Odds ratio adjusted for age and gender. CI: confidence interval; HETE: hydroxyeicosatetraenoic acid; IsoP: isoprostane; Dihydro-15- $\mathrm{F}_{2 \mathrm{t}}$-IsoP: 2,3-dinor-5,6dihydro-15- $\mathrm{F}_{2 \mathrm{t}}$-IsoP; IsoF: isofuran; NeuroP: neuroprostane; HNE: hydroxynonenal; 8-OHdG: 8-hydroxy-2'-deoxy-guanosine.

levels, but high omega- 6 PUFA and saturated fatty acids. The results are consistent with other epidemiological study [5]. Of note, patients who sustained high levels of erythrocyte DHA after DHA supplementation were reported to be significantly protected against AMD compared to those who continued to have low DHA levels (HR 0.32, 95\% CI 0.10-0.99) [27]. The omega-6/omega-3 PUFA ratio of AMD subjects were approximately $35 \%$ higher than the healthy controls in this study. The difference is moderate and likely to be influenced by the dietary habit of the healthy controls who consumed less omega- 6 PUFA food (e.g. red meat) without increasing omega-3 PUFA rich food (e.g. fatty fish) and in AMD subjects, more omega-6 PUFA food and similar amount of omega-3 PUFA food as healthy controls in their meals [19]. Nonetheless, higher plasma omega-6 PUFA levels and lower plasma levels of omega-3 PUFA were found to be risk factors for the development of exudative AMD [19].

Unexpectedly, carotenoid levels were not associated to the disease risk of exudative AMD in the previous study. Although carotenoids (provitamin A) are not potent antioxidants in repressing cellular PUFA 
Table 6

Correlation of nutrition status (total carotenoids and omega-6/omega-3 PUFA ratio) with oxidative damage in vivo (lipid oxidation, DNA oxidation). Only those with statistical significance are shown.

\begin{tabular}{|c|c|c|c|c|c|c|}
\hline & Plasma & & & Urine & & \\
\hline & $\mathrm{r}$ & $\mathrm{n}$ & $p$-value & $\mathrm{r}$ & $\mathrm{n}$ & $p$-value \\
\hline \multicolumn{7}{|l|}{ Total Carotenoids } \\
\hline 5-HETE & -0.123 & 286 & 0.038 & & & \\
\hline 4-HNE & -0.291 & 269 & $<0.0001$ & & & \\
\hline 8-OHdG & -0.161 & 286 & 0.006 & & & \\
\hline \multicolumn{7}{|c|}{ Omega-6/omega-3 PUFA ratio } \\
\hline $15-\mathrm{F}_{2 \mathrm{t}}$-IsoP & 0.138 & 255 & 0.028 & & & \\
\hline Dinor-15- $\mathrm{F}_{2 \mathrm{t}}$-IsoP & & & & 0.125 & 259 & 0.044 \\
\hline 17-dihomo- $\mathrm{F}_{2 \mathrm{t}}$-IsoP & 0.120 & 286 & 0.043 & & & \\
\hline $4-\mathrm{F}_{3 \mathrm{t}}$-IsoP & 0.214 & 286 & $<0.0001$ & 0.161 & 245 & 0.011 \\
\hline $4-\mathrm{F}_{4 \mathrm{t}}-\mathrm{NeuroP}$ & -0.136 & 286 & 0.022 & & & \\
\hline $10-\mathrm{F}_{4 \mathrm{t}}-$ NeuroP & & & & -0.123 & 256 & 0.049 \\
\hline $13-\mathrm{F}_{4 \mathrm{t}}-\mathrm{NeuroP}$ & -0.166 & 275 & 0.006 & & & \\
\hline $14-\mathrm{F}_{4 \mathrm{t}}-$ NeuroP & -0.154 & 275 & 0.010 & & & \\
\hline $20-\mathrm{F}_{4 \mathrm{t}}-\mathrm{NeuroP}$ & -0.205 & 275 & 0.001 & & & \\
\hline RvD1 & -0.140 & 275 & 0.021 & & & \\
\hline 8-OHdG & 0.121 & 286 & 0.041 & & & \\
\hline
\end{tabular}

HETE:hydroxyeicosatetraenoic acid; HNE: hydroxy-nonenal; 8-OHdG: 8-hydroxy-2'-deoxyguanosine; IsoP: isoprostane: NeuroP: neuroprostane; Dinor-15$\mathrm{F}_{2 \mathrm{t}}$-IsoP: 2,3-dinor-5,6-dihydro-15- $\mathrm{F}_{2 \mathrm{t}}$-IsoP; RvD: resolvin D.

oxidation compared to vitamin C and vitamin E, high levels of carotenoids have been constantly reported to be associated with the development of AMD [29], suggesting carotenoids may also take part in regulating in vivo oxidation, including lipid peroxidation, in AMD pathogenesis. Taken together, it is regarded the pathophysiology of AMD risk is due to the resulting metabolites of PUFA oxidation and hence, poor dietary omega-3 PUFA and high omega-6 PUFA may greatly affect the consequence. Therefore, we hypothesized that oxidative damage and low grade inflammation from PUFA oxidation, are partly caused by nutritional status and involved in the development of AMD.

In this study, we determined general in vivo antioxidant-oxidative stress status of the subjects by measuring systemic SOD and GSH and CAT activity. It should be noted, circulating SOD level and catalase activity are not indicators of antioxidant-PUFA oxidation relationship at cellular level. Upregulation of SOD and catalase in this study suggests the AMD patients were under oxidative stress, which matches with a study by Nita and Grzybowski [30]. In addition, plasma levels of GSH were unchanged between groups which also coincides with other report [31]. A positive correlation between SOD and omega-6/omega-3 PUFA ratio was observed in this study and supports the suggestion that high omega-6/omega-3 PUFA ratio to be associated to greater oxidative stress in inflammation [32].

We evaluated a wide panel of PUFA oxidation products from omega6 and omega-3 PUFA, and a DNA oxidation product. The results showed that the plasma levels of omega-6 PUFA oxidation products in the AMD group were significantly higher, including $5-\mathrm{F}_{2 \mathrm{t}^{-}}-\mathrm{IsoP}, 15-\mathrm{F}_{2 \mathrm{t}^{-}}-\mathrm{Iso}, 4-\mathrm{F}_{3 \mathrm{t}^{-}}$ IsoP, 17-dihomo- $\mathrm{F}_{2 \mathrm{t}}$-IsoP, and proinflammatory products 5-HETE and 11-HETE. In addition, elevated urinary $15-\mathrm{F}_{2 \mathrm{t}}$-IsoP and its downstream metabolite 2,3-dinor-5,6-dihydro-15- $\mathrm{F}_{2 \mathrm{t}}$-IsoP were also observed. These findings are consistent with other report but provide additional details on changes in omega-6 PUFA metabolism of AMD [33]. Notably, elevation of urinary 11-HETE strongly increased the odds of AMD development. Although not much is known about 11-HETE in AMD, high levels in circulation was associated with coronary syndrome [34] and lung cancer [35], and take part in neuroinflammation [36] and neurotransmission [37]. In recent report, free 11-HETE was five times higher in obese individuals who had high BMI and waist circumference [38].

Oxidative damage has been implicated as a major contributor in the pathogenesis of AMD. ROS burden in the eye, particularly in the macula, suggests DNA damage to take place. A recent report showed that mitochondrial DNA damage in the retina was associated to AMD. It was found to be localized in the retinal pigment epithelium layer, and in both the macula and peripheral parts [39]. Further, 8-OHdG is one of the most abundant forms of oxidative damage products of DNA and has been associated with ocular diseases such as Grave's ophthalmopathy [40] and found in trabecular meshwork of glaucoma patients [41] and also, augmented in plasma and urine of the AMD patients in this study. Furthermore, it was detected in aqueous humor of exudative AMD patients aged $>55$ years old of Chinese ethnic and were significantly elevated compared to healthy controls [42]. Moreover, in the logistic regression analysis, the oxidation products derived from omega-6 PUFA (5- $\mathrm{F}_{2 \mathrm{t}}$-IsoP, 2,3-dinor-5,6-dihydro-15- $\mathrm{F}_{2 \mathrm{t}}$-IsoP, 17-dihomo- $\mathrm{F}_{2 \mathrm{t}}$-IsoP, $17-$ dihomo- $\mathrm{F}_{2 \mathrm{t}}$-IsoF, 4- $\mathrm{F}_{3 \mathrm{t}}$-IsoP, 4-HNE) and 8-OHdG were associated with a high risk of exudative AMD. Similar to this study, the association between omega-6 PUFA IsoPs and chronic diseases were related to oxidative stress [43]. Nevertheless, $17-$ dihomo- $_{2 t}$-IsoP is considered to be a potential biomarker for neurogenic diseases such as Alzheimer's disease and perhaps, could be the same for exudative AMD [44].

In elevated in vivo oxygen tension (> 21\%, $760 \mathrm{mmHg}$ ), certain PUFAs are oxidized and form isofuranoids [10]. It was found urinary 17-dihomo- $\mathrm{F}_{2 \mathrm{t}}$-IsoF derived from AdA showed high odds in developing AMD. To date, dihomo-IsoF has been identified to be one of the most elevated isofuranoids in the brain tissues of pig and rats [45]. Due to the high content of AdA in the white matter, dihomo-IsoF is considered to be one of the potential biomarkers for lipid peroxidation in the nervous system [21]. It is the very first time that an association has been found between 17 -dihomo- $\mathrm{F}_{2 \mathrm{t}}$-IsoF and neurodegenerative diseases like exudative AMD.

The results from Pearson correlation further explain the relevance of PUFA oxidation in AMD, where high omega-6/omega-3 PUFA ratio and low carotenoids levels were related to the prevalence of omega-6 PUFA oxidation products (mainly 4-HNE, $15-\mathrm{F}_{2 \mathrm{t}}$-IsoP, 2,3-dinor- $15-\mathrm{F}_{2 \mathrm{t}}$-IsoP, 17-dihomo- $\mathrm{F}_{2 \mathrm{t}}$-IsoP, 4- $\mathrm{F}_{3 \mathrm{t}}$-IsoP). In particular, total carotenoid levels were inversely correlated with plasma 4-HNE $(r=-0.291, n=269$, $\mathrm{p}<0.0001$ ). This matches with previous findings in which elevated 4HNE was found in patients with neurodegenerative diseases [46]. The report also points to the role of 4-HNE in pathophysiology of AMD, which has the ability to modify and inactivate retinal proteins by forming adducts $[18,47]$. However, it is unknown if circulating 4-HNE and retinal 4-HNE are proportional, and the likelihood of it being transported to the macula remains unclear.

On the other hand, it was primarily found that plasma $\mathrm{F}_{4 \mathrm{t}}-\mathrm{NeuroPs}$, 14-HDHA, 17-HDHA, RvD1 and NPD1 were down-regulated in the AMD group. To the best of our knowledge, this study is the first attempt to measure metabolism of circulatory omega-3 PUFA in exudative AMD. Increased ROS or oxidative stress typically result in non-specific oxidation of omega- 6 and omega-3 PUFA. Lesser dietary intake of omega-3 PUFA in the AMD subjects compared to healthy controls reported in previous study [19] may partly explain the unexpected reduction of DHA peroxidation products in exudative AMD. Products from the oxidation of omega-3 PUFA (8- $\mathrm{F}_{3 \mathrm{t}}-\mathrm{IsoP}, \mathrm{RvE} 1,4-\mathrm{F}_{4 \mathrm{t}^{-}}$-NeuroP, $10-\mathrm{F}_{4 \mathrm{t}^{-}}$ NeuroP, 20- $\mathrm{F}_{4 \mathrm{t}}$-NeuroP, 14-HDHA, 17-HDHA, NPD1) was also found to be correlated with a lower AMD risk in the regression model. This observation suggests the resolvins and protectins from EPA and DHA take part in the anti-inflammatory and pro-resolving response in AMD [48], thus regulating the low grade inflammation involved in the development of exudative AMD. Furthermore, it is known that NPD1 promotes homeostatic regulation of retinal epithelial cells and photoreceptor cell integrity, particularly during oxidative stress [49]. The reduction in NPD1 level may implicate a decrease in oxidative protection in the macula of AMD patients.

Although the effects of $\mathrm{F}_{4 \mathrm{t}}$-NeuroP on AMD have not previously been studied, recent reports have revealed an increase in 4- $\mathrm{F}_{4 \mathrm{t}}-\mathrm{NeuroP}$ and $10-\mathrm{F}_{4 \mathrm{t}}$-NeuroP in patients with other neurological disorders such as Rett Syndrome [50]. The inconsistency between previous report and our current findings can be explained by the nutritional difference between healthy and AMD groups, in which high omega-6/omega-3 PUFA 
ratio is associated with the down-regulation of omega-3 PUFA oxidation products. Also, previous research reported the level of free $\mathrm{F}_{4}$-NeuroPs while we reported the total (free + esterified) plasma levels. In summary, a low plasma concentration of omega-3 PUFA resulted in decreased bioactive omega-3 PUFA oxidation products level, thus inherently promoted the development of exudative AMD.

Together, results from lipid oxidation product profile indicate patients with exudative AMD have greater oxidative stress in vivo, which leads to oxidative damages, altered lipid metabolism and a higher risk of systemic inflammation. A high omega-6/omega-3 PUFA ratio and low carotenoid levels lead to the prevalence of omega-6 PUFA metabolites, as well as down-regulation of anti-inflammatory and pro-resolving omega-3 PUFA oxidation products. All of these factors contribute to the development of exudative AMD. Notably, the measurement of circulating PUFA oxidation products only relatively reflects changes in systemic PUFA metabolism and the condition may differ from that of target tissue in AMD (i.e. retinal pigment epithelial cells). Nonetheless, current research enhances our understanding of how nutrition regulates the pathogenesis of exudative AMD in the molecular level through lipid metabolism modulation. It also provides a context for future prospective studies on the use of carotenoids and omega-3 PUFA in AMD prevention and regulation.

\section{Funding}

This work was supported by Health and Medical Research Fund (Ref: 13142301), Food and Health Bureau, Hong Kong.

\section{Appendix A. Supplementary data}

Supplementary data to this article can be found online at

\section{References}

[1] W.M. Al-Zamil, S.A. Yassin, Recent developments in age-related macular degeneration: a review, Clin. Interv. Aging 12 (2017) 1313-1330.

[2] A. Kijlstra, T.T. Berendschot, Age-related macular degeneration: a complementopathy? Ophthalmic Res. 54 (2) (2015) 64-73.

[3] G. Age-Related Eye Disease Study Research, A randomized, placebo-controlled, clinical trial of high-dose supplementation with vitamins C and E, beta carotene, and zinc for age-related macular degeneration and vision loss: AREDS report no. 8, Arch. Ophthalmol. 119 (10) (2001) 1417-1436.

[4] G. Age-Related Eye Disease Study 2 Research, Lutein + zeaxanthin and omega-3 fatty acids for age-related macular degeneration: the Age-Related Eye Disease Study 2 (AREDS2) randomized clinical trial, J. Am. Med. Assoc. 309 (19) (2013) 2005-2015.

[5] E.H. Souied, T. Aslam, A. Garcia-Layana, F.G. Holz, A. Leys, R. Silva, C. Delcourt, Omega-3 fatty acids and age-related macular degeneration, Ophthalmic Res. 55 (2) (2015) 62-69.

[6] J.W. Crabb, M. Miyagi, X. Gu, K. Shadrach, K.A. West, H. Sakaguchi, M. Kamei, A. Hasan, L. Yan, M.E. Rayborn, R.G. Salomon, J.G. Hollyfield, Drusen proteome analysis: an approach to the etiology of age-related macular degeneration, Proc. Natl. Acad. Sci. U. S. A. 99 (23) (2002) 14682-14687.

[7] B. Farboud, A. Aotaki-Keen, T. Miyata, L.M. Hjelmeland, J.T. Handa, Development of a polyclonal antibody with broad epitope specificity for advanced glycation endproducts and localization of these epitopes in Bruch's membrane of the aging eye, Mol. Vis. 5 (1999) 11.

[8] S. Beatty, H. Koh, M. Phil, D. Henson, M. Boulton, The role of oxidative stress in the pathogenesis of age-related macular degeneration, Surv. Ophthalmol. 45 (2) (2000) 115-134.

[9] F.L. Muller, M.S. Lustgarten, Y. Jang, A. Richardson, H. Van Remmen, Trends in oxidative aging theories, Free Radic. Biol. Med. 43 (4) (2007) 477-503.

[10] J.M. Galano, J.C. Lee, C. Gladine, B. Comte, J.Y. Le Guennec, C. Oger, T. Durand, Non-enzymatic cyclic oxygenated metabolites of adrenic, docosahexaenoic, eicosapentaenoic and alpha-linolenic acids; bioactivities and potential use as biomarkers, Biochim. Biophys. Acta 1851 (4) (2015) 446-455.

[11] L.J. Roberts, J.D. Morrow, Measurement of F(2)-isoprostanes as an index of oxidative stress in vivo, Free Radic. Biol. Med. 28 (4) (2000) 505-513.

[12] J. Bauer, A. Ripperger, S. Frantz, S. Ergun, E. Schwedhelm, R.A. Benndorf, Pathophysiology of isoprostanes in the cardiovascular system: implications of isoprostane-mediated thromboxane A2 receptor activation, Br. J. Pharmacol. 171 (13) (2014) 3115-3131.

[13] L.J. Janssen, The pulmonary biology of isoprostanes, Chem. Phys. Lipids 128 (1-2) 2004) 101-116.
[14] J. Roy, J. Fauconnier, C. Oger, C. Farah, C. Angebault-Prouteau, J. Thireau, P. Bideaux, V. Scheuermann, V. Bultel-Ponce, M. Demion, J.M. Galano, T. Durand, J.C. Lee, J.Y. Le Guennec, Non-enzymatic oxidized metabolite of DHA, 4(RS)-4-F4tneuroprostane protects the heart against reperfusion injury, Free Radic. Biol. Med. 102 (2017) 229-239.

[15] C. Signorini, C. De Felice, T. Durand, J.M. Galano, C. Oger, S. Leoncini, L. Ciccoli, M. Carone, M. Ulivelli, C. Manna, A. Cortelazzo, J.C. Lee, J. Hayek, Relevance of 4 F4t-neuroprostane and 10-F4t-neuroprostane to neurological diseases, Free Radic. Biol. Med. 115 (2018) 278-287.

[16] K.N. Ikei, J. Yeung, P.L. Apopa, J. Ceja, J. Vesci, T.R. Holman, M. Holinstat, Investigations of human platelet-type 12-lipoxygenase: role of lipoxygenase products in platelet activation, J. Lipid Res. 53 (12) (2012) 2546-2559.

[17] J. Kopitz, F.G. Holz, E. Kaemmerer, F. Schutt, Lipids and lipid peroxidation products in the pathogenesis of age-related macular degeneration, Biochimie 86 (11) (2004) 825-831.

[18] M. Shoeb, N.H. Ansari, S.K. Srivastava, K.V. Ramana, 4-Hydroxynonenal in the pathogenesis and progression of human diseases, Curr. Med. Chem. 21 (2) (2014) 230-237

[19] A.L. Ng, H.H. Leung, R. Kawasaki, W.L. Ho, L.L. Chow, S.S. Chow, J.C. Lee, I.Y. Wong, Dietary habits, fatty acids and carotenoid levels are associated with neovascular age-related macular degeneration in Chinese, Nutrients 11 (8) (2019).

[20] A. de la Torre, Y.Y. Lee, A. Mazzoni, A. Guy, V. Bultel-Ponce, T. Durand, C. Oger, J.C. Lee, J.M. Galano, Total syntheses and in vivo quantitation of novel neurofuran and dihomo-isofuran derived from docosahexaenoic acid and adrenic acid, Chemistry 21 (6) (2015) 2442-2446.

[21] A. de La Torre, Y.Y. Lee, C. Oger, P.T. Sangild, T. Durand, J.C. Lee, J.M. Galano, Synthesis, discovery, and quantitation of dihomo-isofurans: biomarkers for in vivo adrenic acid peroxidation, Angew Chem. Int. Ed. Engl. 53 (24) (2014) 6249-6252.

[22] C. Oger, V. Bultel-Ponce, A. Guy, L. Balas, J.C. Rossi, T. Durand, J.M. Galano, The handy use of Brown's P2-Ni catalyst for a skipped diyne deuteration: application to the synthesis of a [D4]-labeled F4t-neuroprostane, Chemistry 16 (47) (2010) 13976-13980.

[23] A. Guy, C. Oger, J. Heppekausen, C. Signorini, C. De Felice, A. Furstner, T. Durand, J.M. Galano, Oxygenated metabolites of n-3 polyunsaturated fatty acids as potential oxidative stress biomarkers: total synthesis of 8-F3t-IsoP, 10-F4t-NeuroP and [D4] 10-F4t-NeuroP, Chemistry 20 (21) (2014) 6374-6380.

[24] G. Dayaker, T. Durand, L. Balas, Total synthesis of neuroprotectin D1 analogues derived from omega- 6 docosapentaenoic acid (DPA) and adrenic acid (AdA) from a common pivotal, late-stage intermediate, J. Org. Chem. 79 (6) (2014) 2657-2665.

[25] Y.Y. Lee, J.C. Lee, LC-MS/MS analysis of lipid oxidation products in blood and tissue samples, Methods Mol. Biol. 1730 (2018) 83-92.

[26] C.W. Hu, M.T. Wu, M.R. Chao, C.H. Pan, C.J. Wang, J.A. Swenberg, K.Y. Wu, Comparison of analyses of urinary 8-hydroxy-2'-deoxyguanosine by isotope-dilution liquid chromatography with electrospray tandem mass spectrometry and by enzyme-linked immunosorbent assay, Rapid Commun. Mass Spectrom. 18 (4) (2004) 505-510

[27] E.H. Souied, C. Delcourt, G. Querques, A. Bassols, B. Merle, A. Zourdani, T. Smith, P. BenlianA.M.D.T.S.G. Nutritional, Oral docosahexaenoic acid in the prevention of exudative age-related macular degeneration: the Nutritional AMD Treatment 2 study, Ophthalmology 120 (8) (2013) 1619-1631.

[28] B.M. Merle, P. Benlian, N. Puche, A. Bassols, C. Delcourt, E.H. SouiedA.M.D.T.S.G. Nutritional, Circulating omega-3 Fatty acids and neovascular age-related macular degeneration, Investig. Ophthalmol. Vis. Sci. 55 (3) (2014) 2010-2019.

[29] J. Wu, E. Cho, W.C. Willett, S.M. Sastry, D.A. Schaumberg, Intakes of lutein, zeaxanthin, and other carotenoids and age-related macular degeneration during 2 decades of prospective follow-up, JAMA Ophthalmol 133 (12) (2015) 1415-1424.

[30] M. Nita, A. Grzybowski, The role of the reactive oxygen species and oxidative stress in the pathomechanism of the age-related ocular diseases and other pathologies of the anterior and posterior eye segments in adults, Oxid Med Cell Longev (2016) (2016) 3164734.

[31] Z. Yildirim, N.I. Ucgun, F. Yildirim, The role of oxidative stress and antioxidants in the pathogenesis of age-related macular degeneration, Clinics (Sao Paulo) 66 (5) (2011) 743-746.

[32] J.J. DiNicolantonio, J.H. O'Keefe, Importance of maintaining a low omega-6/ omega-3 ratio for reducing inflammation, Open Heart 5 (2) (2018) e000946.

[33] C. Sabanayagam, W.K. Lye, A. Januszewski, R. Banu Binte Mohammed Abdul, G.C.M. Cheung, N. Kumari, T.Y. Wong, C.Y. Cheng, E. Lamoureux, Urinary isoprostane levels and age-related macular degeneration, Investig. Ophthalmol. Vis Sci. 58 (5) (2017) 2538-2543.

[34] L. Zu, G. Guo, B. Zhou, W. Gao, Relationship between metabolites of arachidonic acid and prognosis in patients with acute coronary syndrome, Thromb. Res. 144 (2016) 192-201.

[35] J. Liu, P.J. Mazzone, J.P. Cata, A. Kurz, M. Bauer, E.J. Mascha, D.I. Sessler, Serum free fatty acid biomarkers of lung cancer, Chest 146 (3) (2014) 670-679.

[36] G. Hayashi, Y. Shen, T.L. Pedersen, J.W. Newman, M. Pook, G. Cortopassi, Frataxin deficiency increases cyclooxygenase 2 and prostaglandins in cell and animal models of Friedreich's ataxia, Hum. Mol. Genet. 23 (25) (2014) 6838-6847.

[37] X. Yu, J. Wu, M. Hu, J. Wu, Q. Zhu, Z. Yang, X. Xie, Y.Q. Feng, J. Yue, Glutamate affects the CYP1B1- and CYP2U1-mediated hydroxylation of arachidonic acid metabolism via astrocytic mGlu5 receptor, Int. J. Biochem. Cell Biol. 110 (2019) 111-121.

[38] C.A. Pickens, L.M. Sordillo, C. Zhang, J.I. Fenton, Obesity is positively associated with arachidonic acid-derived 5- and 11-hydroxyeicosatetraenoic acid (HETE), Metabolism 70 (2017) 177-191.

[39] M.R. Terluk, R.J. Kapphahn, L.M. Soukup, H. Gong, C. Gallardo, S.R. Montezuma, D.A. Ferrington, Investigating mitochondria as a target for treating age-related 
macular degeneration, J. Neurosci. 35 (18) (2015) 7304-7311.

[40] C.C. Tsai, S.C. Kao, C.Y. Cheng, H.C. Kau, W.M. Hsu, C.F. Lee, Y.H. Wei, Oxidative stress change by systemic corticosteroid treatment among patients having active graves ophthalmopathy, Arch. Ophthalmol. 125 (12) (2007) 1652-1656.

[41] H.C. Kau, C.C. Tsai, C.F. Lee, S.C. Kao, W.M. Hsu, J.H. Liu, Y.H. Wei, Increased oxidative DNA damage, 8-hydroxydeoxy- guanosine, in human pterygium, Eye (Lond) 20 (7) (2006) 826-831.

[42] L.I. Lau, C.J. Liu, Y.H. Wei, Increase of 8-hydroxy-2'-deoxyguanosine in aqueous humor of patients with exudative age-related macular degeneration, Investig. Ophthalmol. Vis. Sci. 51 (11) (2010) 5486-5490.

[43] A. Garcia-Blanco, C. Pena-Bautista, C. Oger, C. Vigor, J.M. Galano, T. Durand, N. Martin-Ibanez, M. Baquero, M. Vento, C. Chafer-Pericas, Reliable determination of new lipid peroxidation compounds as potential early Alzheimer Disease biomarkers, Talanta 184 (2018) 193-201.

[44] M. VanRollins, R.L. Woltjer, H. Yin, J.D. Morrow, T.J. Montine, F2-dihomo-isoprostanes arise from free radical attack on adrenic acid, J. Lipid Res. 49 (5) (2008) 995-1005.
[45] D.F. Taber, J.P. Fessel, L.J. Roberts, 2nd, A nomenclature system for isofurans, Prostaglandins Other Lipid Mediat. 73 (1-2) (2004) 47-50.

[46] M. Csala, T. Kardon, B. Legeza, B. Lizak, J. Mandl, E. Margittai, F. Puskas, P. Szaraz, P. Szelenyi, G. Banhegyi, On the role of 4-hydroxynonenal in health and disease, Biochim. Biophys. Acta 1852 (5) (2015) 826-838.

[47] C.M. Ethen, C. Reilly, X. Feng, T.W. Olsen, D.A. Ferrington, Age-related macular degeneration and retinal protein modification by 4-hydroxy-2-nonenal, Investig. Ophthalmol. Vis. Sci. 48 (8) (2007) 3469-3479.

[48] C.N. Serhan, N. Chiang, T.E. Van Dyke, Resolving inflammation: dual anti-inflammatory and pro-resolution lipid mediators, Nat. Rev. Immunol. 8 (5) (2008) 349-361.

[49] N.G. Bazan, Neuroprotectin D1-mediated anti-inflammatory and survival signaling in stroke, retinal degenerations, and Alzheimer's disease, J. Lipid Res. 50 (Suppl) (2009) S400-S405.

[50] C. Signorini, C. De Felice, J.M. Galano, C. Oger, S. Leoncini, A. Cortelazzo, L. Ciccoli, T. Durand, J. Hayek, J.C. Lee, Isoprostanoids in clinical and experimental neurological disease models, Antioxidants 7 (7) (2018). 